\title{
O IMPACTO DA CULTURA NO USO DE PRODUTOS COM TECNOLOGIA AGREGADA: ESTUDO COM ADOLESCENTES
}

DOI: 1014211/regepe.v5i1.316

Artigo recebido em:26/09/2015 Artigo aprovado em:08/03/2016

\author{
Andressa Gonçalves - Senac Blumenau ${ }^{1}$ \\ Silvana Vieira Tambosi- Universidade Regional de Blumenau ${ }^{2}$ \\ Vanessa Mondini - Universidade Regional de Blumenau ${ }^{3}$ \\ Denise Del Pra Netto Machado - Universidade Regional de Blumenau ${ }^{4}$
}

Resumo: Esta pesquisa teve como objetivo identificar elementos da cultura que podem influenciar na adoção de inovações tecnológicas por adolescentes do Vale do Itajaí. A abordagem foi quantitativa, com o uso da técnica estatística de Regressão Linear Múltipla. A coleta de dados foi feita com aplicação de 472 questionários. Os resultados indicaram que os adolescentes tendem a confrontar mais as decisões de seus pais, e apresentaram uma tendência ao individualismo e à baixa distância do poder. Ressalta-se que o aspecto medido nesta pesquisa se refere a adoção de inovações tecnológicas e os relacionamentos com os pais e colegas.

Palavras-chave: Cultura; Inovações Tecnológicas; Adolescentes.

\section{THE IMPACT OF CULTURE ON THE USE OF PRODUCTS WITH ADDED TECHNOLOGY: A STUDY ON ADOLESCENTS}

\begin{abstract}
This research aimed to identify elements of culture that can influence the adoption of technological innovations by teenagers of Vale of Itajaí. The approach was quantitative, using the statistical technique of multiple linear regression. The data collection was made with application of 472 questionnaires. The results indicated that adolescents tend to face more decisions of their parents, and showed a tendency to individualism and low power distance. It should be noted that the aspect measured in this study refers to adoption of technological innovations and relationships with parents and colleagues.
\end{abstract}

Keywords: Culture; Technological Innovations; Adolescents.

\footnotetext{
E-mail: goncalves.dessaa@gmail.com

${ }^{2}$ Endereço: R. Antônio da Veiga, 140 - Itoupava Seca, Blumenau - SC, 89012-900E-mail: profa.silvana.vieira@gmail.com

${ }^{3}$ E-mail: prof.vanessa@ymail.com

${ }^{4}$ E-mail: profadenisedellpra@gmail.com
}

GONÇALVES, A.; TAMBOSI, S. V.; MONDINI, V.; MACHADO, D. D. P. N.O impacto da cultura no uso de produtos com tecnologia agregada: estudo com adolescentes. Revista de Empreendedorismo e Gestão de Pequenas Empresas, v.5, n.1, 2016. 


\section{Introdução}

A inovação é considerada um tema importante para várias áreas do saber, tais como: economia, negócios, engenharia, ciência e sociologia. Por definição, todas as inovações têm um elemento de novidade, pois são diferentes do que existia antes. Assim, a inovação é o resultado de um processo que envolve a utilização de uma nova abordagem para a criação ou comercialização de produtos ou serviços.

Desta forma, entende-se que a inovação está diretamente relacionada com o desenvolvimento de uma nova ideia, invenção, método ou dispositivo aplicado em um processo para a introdução de algo novo que tenha aplicação no mercado (GOPALAKRISHNANAN; DAMANPOUR, 1997; AFUAH, 2003; CHANDY; PRABHU, 2010).

Vale destacar que, neste estudo, será utilizada a abordagem de inovação como resultado de um processo. Ao abordar este tema, questões relacionadas à tecnologia geralmente entram em pauta. Novos celulares, tablets, aplicativos, computadores e jogos eletrônicos surgem e estão em ascensão nas mídias sociais dos jovens e adolescentes, e diante deste cenário, o público adolescente ocupa lugar de destaque.

Extremamente conectados, estes adolescentes nascidos a partir da década de 1990, são rotulados como nativos digitais pela sua ampla familiaridade com a tecnologia. Este segmento, no entanto, é caracterizado por jovens que, em sua maioria, ainda moram com os pais e estão sujeitos ao compartilhamento de crenças, normas e regras familiares que podem influenciar nas suas decisões (MCCRINDLE, 2011).

Mesmo que nesta faixa etária os jovens busquem uma independência de opinião em relação a dos pais, a família ainda exerce um forte impacto em suas decisões. Uma parcela expressiva de influência também é exercida pelos colegas. Ao interagirem com o grupo, preferências, críticas e formas de uso de produtos inovadores emergem impactando na avaliação de compra para si (MCCRINDLE, 2011). 
Sob esta perspectiva, o presente artigo apresenta a seguinte questão de pesquisa: Quais os elementos da cultura, representados pela distância do poder e coletivismo versus individualismo podem influenciar na adoção de inovações que possuem tecnologia agregada por adolescentes?

Desta maneira, objetiva-se identificar elementos da cultura por meio das dimensões distância do poder e coletivismo versus individualismo, que podem influenciar na adoção de inovações tecnológicas por adolescentes de uma escola particular no Vale do Itajaí.

O texto está estruturado em quatro seções além desta introdução. Em seguida, será apresentado o referencial teórico que servirá de base para a discussão dos resultados. A terceira parte contém os aspectos metodológicos e a caracterização da população de pesquisa. E, por fim, exibem-se a discussão dos resultados e as considerações finais, respectivamente.

\section{Inovação}

O termo inovação recebe inúmeras definições, podendo ser caracterizado como um novo produto ou serviço, uma nova tecnologia de processo ou de produção, um sistema administrativo ou uma nova estrutura organizacional. A inovação é considerada uma das estratégias de crescimento fundamentais da organização para entrar em novos mercados ou aumentar a quota existente e para fornecer à empresa uma vantagem competitiva (DAMANPOUR, 1992).

Schumpeter (1934), um dos autores mais citados quando a temática é inovação, enfatiza que o termo não é relacionado apenas a produtos e processos, mas também ao marketing e à forma de gestão de uma organização. O autor afirma que a inovação pode acontecer em produtos, métodos de produção, fontes de abastecimento, exploração de novos mercados e em novas formas de organização.

A noção de que existem diferentes tipos de inovação com diferentes efeitos sobre a concorrência, é um tema importante na literatura sobre inovação tecnológica desde Schumpeter (1934), que caracterizou os diferentes tipos de inovações em 
termos de seu impacto sobre as capacidades estabelecidas da empresa (HENDERSON; CLARK, 1990).

Em virtude das inúmeras definições presentes na literatura, a Organização para Cooperação e Desenvolvimento Econômico (OECD) desenvolveu um documento chamado Manual de Oslo, buscando uma uniformidade no conceito de inovação. Este manual contém um conjunto de conceitos e classificações da inovação em âmbito internacional (OECD, 2005).

No Manual de Oslo da OECD (2005) são apresentados quatro tipos de inovação. Trata-se de inovação de produto, processo, de marketing e organizacional. As inovações de produtos e processos estão intimamente relacionadas com o conceito de desenvolvimento tecnológico, sendo caracterizadas pela introdução de um serviço que é novo ou significativamente aprimorado em relação às suas características iniciais, incluindo melhorias em especificações técnicas, componentes e materiais, facilidade de uso ou outras características funcionais.

A inovação de produto, a ser trabalhada com maior ênfase neste estudo, é um processo impulsionado pelo avanço de tecnologias, mudanças nas necessidades dos clientes, reduzindo os ciclos de vida dos produtos e aumento da concorrência global. Para o sucesso, este tipo de inovação deve envolver alta interação entre ambiente interno e externo à organização, principalmente com clientes e fornecedores (AKOVA et al., 1998).

A inovação tecnológica consiste na criação de um novo produto ou em modificações nos atributos de produtos existentes, com mudanças na forma de como ele é percebido pelos consumidores. Neste sentido, a inovação tecnológica é uma força poderosa para o desenvolvimento industrial, para o crescimento da produtividade e como padrão do nível de maturidade das organizações (TUSHMAN et al., 1992).

Tushman et al. (1992) descrevem o ciclo de vida da tecnologia e a sua evolução, constituído por quatro etapas: i) uma descontinuidade tecnológica, que ocorre por meio da variação ou da adoção de nova tecnologia; ii) período de fermentação tecnológica e competitividade, no qual ocorre uma competição entre projetos variantes, que irão competir com a nova tecnologia; iii) período de seleção 
do projeto dominante que irá adquirir a forma de um novo produto ou com novas características; e iv) processo de substituição em relação à tecnologia antiga, ou seja, incrementos no produto ou no processo já existente (TUSHMAN et al., 1992).

No ambiente competitivo contemporâneo, a inovação de produtos está se tornando cada vez mais relevante, principalmente como resultado de três grandes tendências: uma intensa concorrência internacional, mercados fragmentados e exigentes, e a constante evolução tecnológica (COOPER; KLEINSCHMIDT, 1993).

As empresas que oferecem produtos adaptados às necessidades e desejos dos clientes-alvo de forma mais rápida e eficiente do que seus concorrentes estão em melhor posição e com condições favoráveis para criarem uma vantagem competitiva sustentável, tendo em vista que esta vantagem deriva cada vez mais de conhecimentos e habilidades tecnológicas e experiências na criação de novos produtos (ALEGRE; LAPIEDRA; CHIVA, 2006).

Segundo Cooper e Kleinschmidt (1993), Brown e Eisenhardt (1995), Griffin (1997) e Alegre, Lapiedra e Chiva (2006), o desenvolvimento do produto é fundamental para a viabilidade das empresas. A gestão bem estruturada do processo de desenvolvimento de novos produtos pode significar maior capacidade de diversificação, potencial para a transformação de novas tecnologias em novos produtos, melhores parcerias e menores custos, além de ser um meio fundamental no qual os membros das organizações conseguem diversificar, adaptar e até reinventar suas empresas para combinar com a evolução das condições de mercado.

Como apresentado até aqui, a inovação refere-se à capacidade da empresa no desenvolvimento de novos produtos ou serviços, bem como a habilidade que as organizações possuem, juntamente com seus recursos e o seu conhecimento técnico, para desenvolver e criar novas oportunidades no mercado.

Nesta pesquisa, o objeto de estudo não será a inovação empresarial, mas sim, a adoção de produtos inovadores por parte de estudantes de uma escola técnica. Partindo da ideia de que as organizações desenvolvem os produtos inovadores, o estudo irá tratar da capacidade de absorção desses produtos pelos consumidores que, nesta pesquisa, fazem parte os adolescentes de uma escola do Vale do Itajaí. A absorção das tecnologias produzidas pelas empresas por parte dos 
adolescentes pertencentes a esta amostra será tratada pelo termo inovatividade, conforme apresentado a seguir.

\section{Inovatividade}

A inovatividade é a capacidade inovativa da empresa em criar "novidade", ou seja, está relacionada com a habilidade em desenvolver e lançar novos produtos em um ritmo rápido (HURLEY; HULT, 1998). A inovatividade está diretamente ligada aos indivíduos que representam a organização na criação desses produtos/serviços inovadores. Já o conceito de consumidor inovativo refere-se à tendência das pessoas em comprar novos produtos com mais frequência e mais rapidamente do que outras pessoas, está ligado à capacidade inovativa pessoal (MIDGLEY; DOWLING, 1978).

Neste estudo, assim como nos estudos de Roehrich (2004), o conceito de inovatividade está relacionado unicamente com a capacidade de absorver inovação por parte do consumidor.

A teoria caracteriza a capacidade inovativa do consumidor com o termo "inato", que é explicada como sendo uma característica inerente ao ato de consumir em relação à inovatividade. Essa característica é abordada em várias pesquisas empíricas que reforçam esse traço do indivíduo pré-disposto a adoção da inovação (HURT; JOSEPH; COOK, 1977; CLARK; GOLDSMITH, 2006; CHAO; REID; MAVONDO, 2009).

Steenkamp, Hofstede e Wedel (1999) reforçam que inovatividade é uma predisposição que o consumidor tem em adquirir novos e diferentes produtos em vez de permanecer com as escolhas e padrões de consumo anteriores. Os autores explicam essa predisposição com base em quatro fatores, são eles: (a) necessidade de estímulo; (b) busca de novidade; (c) independência com relação à experiência com outros meios de comunicação; e (d) necessidade de singularidade (único).

Estudos sobre a inovatividade são importantes ferramentas para as organizações medirem e preverem o comportamento de compra dos consumidores. Este viés foi utilizado nos estudos de Goldsmith e Hofacker (1991) e Agarwal e 
Prasad (1998) para definir o termo "inovatividade pessoal" no domínio das tecnologias de informação (PIIT).

Os autores, Goldsmith e Hofacker (1991) conferem esse termo à propensão de um indivíduo em experimentar uma nova tecnologia de informação. Ao identificar os indivíduos mais predispostos a experimentar uma nova tecnologia, seria possível permitir que eles atuassem como agentes de mudança e líderes de opinião para novas implementações de tecnologia de informação em ambientes organizacionais.

Nessa mesma linha de considerações, Eastlick e Lotz (1999) desenvolveram um modelo teórico que relacionava os traços de inovatividade pessoal, como: liderança opinião/inovação, busca de informações, com as intenções de adoção de novas tecnologias, através de uma construção de atitude. Como resultado, verificaram, em um estudo centrado em comércio eletrônico, que os indivíduos com maior pontuação na escala liderança/inovadora eram mais propensos à intenção de comprar produtos inovadores do que aqueles que obtiveram pontuação mais baixa nessa mesma escala.

Daghfous, Petrof e Pons (1999) afirmam que a adoção de inovações sofre o crivo do sistema de valores do indivíduo. Dispostos a estudar a incidência de valores culturais sobre a inclinação de indivíduo a adotarem inovações, estes autores realizaram uma investigação multicultural com consumidores provenientes de três grupos culturais distintos: os alunos da escola de negócios de Quebec, França e Norte da África.

Os resultados obtidos indicaram que os valores individuais impactam significativamente na propensão dos consumidores em adotar inovações. Para os autores, em se tratando de mercados heterogêneos e multiculturais, a segmentação dos consumidores em função dos seus valores é uma importante ferramenta estratégica de marketing.

Tolba e Mourad (2011), por sua vez, afirmam que indivíduos tidos como líderes de opinião são percebidos por seus seguidores como: possuidores de nível socioeconômico mais elevado; um modelo a ser copiado; e com maior predisposição a adotar ideias inovadoras. Suas principais características são: o conhecimento a capacidade de persuasão e a inovação. Para Rosen (2005), estes líderes de opinião possuem maior capacidade de inovatividade pessoal e sofrem influências de cultura no uso de produtos com tecnologia agregada: estudo com adolescentes. Revista de Empreendedorismo e Gestão de Pequenas Empresas, v.5, n.1, 2016. 
variáveis culturais, como: atitudes, normas subjetivas e controle comportamental das pessoas.

Para avaliar a dimensão de Inovatividade e posteriormente relacioná-la à Cultura, será usado o modelo proposto por Parasuramann (2000), cujas questões se referem à posição do respondente com relação: a conhecimentos sobre novidades tecnológicas; se o respondente serve de referência para os colegas quando o assunto é novidade tecnológica; se acha que tem menos problemas do que outras pessoas em usar novidades; se já evitou experimentar novidades tecnológicas; ou se acha que não há sentido em experimentar tais novidades.

\section{Cultura}

A cultura é um conjunto de significados aceitos por um grupo em um determinado momento (PETTIGREW, 1979). Nesta perspectiva, a cultura estabelece um cenário cujas normas, sobre o que é certo e errado, direcionam a conduta dos indivíduos e os levam a interagirem socialmente de forma adequada. Desta maneira, a cultura pode influenciar na forma de comunicação e compartilhamento de conhecimentos e experiências entre as pessoas. De maneira mais específica, Hofstede (1980), a partir de estudos sobre cultura, realizados em 50 países, definiu quatro dimensões denominadas de: Distância do Poder, Coletivismo versus Individualismo, Feminilidade versus Masculinidade e Controle da Incerteza.

Para atender ao objetivo deste estudo, que é identificar elementos da cultura na adoção de inovatividade por adolescentes quando o assunto é o uso de novas tecnologias, elegeu-se duas das dimensões propostas nos estudos de Hofstede (1980): individualismo/coletivismo e distância do poder. De acordo com Parasuramann (2000), esta escolha se deu em função do entendimento de que tanto a relação com os grupos sociais quanto a relação entre pais e filhos influenciam na inovatividade do adolescente.

A dimensão "individualismo e coletivismo" se refere ao nível de adesão das pessoas aos grupos sociais. Modelos culturais representados pelo individualismo e coletivismo levam os sujeitos a entenderem o mundo por meio de diferentes pontos 
de vista e a interpretarem os eventos sociais sob óticas distintas. Culturas individualistas são formadas por indivíduos mais autônomos e independentes em relação aos seus grupos sociais. Já culturas coletivistas se compõem de sujeitos mais dependentes dos valores dos grupos aos quais pertencem (TRIANDIS, 1995).

Segundo Gouveia, Clemente e Vidal (1998), o sujeito individualista valoriza a adesão aos grupos em função de prováveis benefícios. Este perfil sugere que há uma independência em relação aos familiares e uma desvinculação dos laços parentais. Pessoas guiadas pelo individualismo tendem a restringir seus grupos apenas à1 família nuclear. Suas ações e pensamentos se voltam primeiramente aos interesses próprios, relegando a um segundo plano, o contexto social no qual se encontra (GOUVEIA, 2001). Culturas individualistas se caracterizam pela influência de crenças pessoais na tomada de decisões e nem sempre as regras do grupo são rigorosamente seguidas (PEREZ-ALVAREZ, 2009).

Já no coletivismo, as relações são estabelecidas em função do compartilhamento de interesses comuns. Existe uma tendência à colaboração tornando o grupo do qual a pessoa participa mais importante do que o indivíduo. Sujeitos coletivistas tendem a considerar toda a família, incluindo ascendentes e descendentes, como parte do seu grupo. Conflitos são indesejados e o indivíduo atua levando em consideração o bem-estar do grupo (GOUVEIA, 2001).

Acredita-se que indivíduos que vivem em sociedades coletivistas são mais influenciados pela sociedade em relação a suas decisões de adotar inovações do que aqueles que vivem em culturas individualistas. Conforme Triandis (1995), um tipo ou outro de orientação cultural levará os indivíduos a comportamentos distintos, tanto a respeito do que pensam sobre si próprios, quanto em relação as suas posturas perante aos grupos sociais.

Dispostos a desenvolver um modelo conceitual que incorporasse a aceitação e difusão da inovação, Tolba e Mourad (2011) verificaram que a cultura exerce um papel expressivo no processo de difusão da inovação. Para os autores, em culturas individualistas, as inovações demoram mais para serem aceitas, do que em culturas coletivistas. Por outro lado, após aceitas, a difusão destas inovações é mais rápida em culturas individualistas do que nas coletivistas. 
Para avaliar esta dimensão do "individualismo e coletivismo", será usado o modelo proposto por Hofstede (1980), cujas questões estão relacionadas a: possibilidade de renúncia de objetivos e interesses próprios em favor do grupo, permanência no grupo mesmo quando ele apresentar dificuldades e prevalência do bem-estar do grupo sobre as perspectivas de recompensas individuais.

A dimensão "distância do poder", por sua vez, reflete o grau de aceitação dos membros menos poderosos das organizações sobre uma distribuição assimétrica do poder. Encontram-se categoria, instituições, tais como: a família, a escola e a comunidade (HOFSTEDE, 1980). Ainda com relação a esta dimensão, o autor afirma que uma distância hierárquica pequena tende a reduzir as desigualdades entre as pessoas.

Especialmente no ambiente de convívio dos adolescentes, sob esta perspectiva de distância curta do poder, pais e filhos tratam-se como iguais e os professores são vistos como líderes. Já em culturas com grande distância do poder, os pais educam os filhos para a obediência. Os filhos, por sua vez, tratam os pais de forma mais respeitosa e os professores são percebidos por eles como especialistas impessoais (HOFSTEDE, 1980).

Kalliny e Hausman (2007) estudaram o impacto das culturas de diferentes países na adoção de inovação e verificaram que, em culturas com pouca distância do poder, como a americana, a influência de grupos de aspiração impacta positivamente na adoção de inovação por parte dos consumidores, que os veem como um modelo a seguir. Já em sociedades com alta distância do poder, como a islâmica, os consumidores são desencorajados a imitar quem está no poder, inclusive por meio de proibição de consumo de novidades.

Esta dimensão "distância do poder", será avaliada também pelo modelo de Hofstede (1980), por meio de questões que envolvem o entendimento do adolescente a respeito: da concentração da tomada de decisão na figura dos pais; aceitação destas decisões; e posicionamento da família sobre a possibilidade de envolvimento com pessoas de outra classe social. 


\section{Procedimentos Metodológicos}

Para responder à pergunta de pesquisa, utilizou-se o estudo descritivo com corte transversal. De acordo com Hair Jr. et al. (2009), a pesquisa descritiva normalmente detalha alguma situação, sendo estruturada e criada especialmente para mensurar as características de eventos ou atividades de pesquisa. Assim, optou-se pelo corte transversal com survey, por proporcionar a coleta dos dados em determinado espaço de tempo e a sintetização estatística destes dados (HAIR Jr. et al., 2009).

A população desta pesquisa foi composta por 493 alunos do Ensino Médio, formado por turmas de primeiro, segundo e terceiros anos de uma Escola Técnica situada no Vale do Itajaí, com idade entre 14 e 18 anos, pertencente a uma Universidade. A escola está situada dentro dos domínios da Universidade e os alunos do Ensino Médio convivem com alunos do Ensino Superior nos períodos matutino e vespertino. Do total de alunos, 472 responderam aos questionários, representando 95,74\% da população, aplicados em sala de aula, nos dias 20 e 21 de fevereiro de 2014.

No tocante ao instrumento utilizado para a coleta de dados, optou-se pelo questionário adaptado de Hofstede (1980), relativo às dimensões da cultura. Este instrumento é dividido em duas dimensões, sendo elas: distância do poder e coletivismo versus individualismo. Utilizou-se ainda o questionário de Parasuramann (2000), referente à inovatividade, sendo composto por uma dimensão com questões referentes ao uso de tecnologias e produtos inovadores.

As questões das dimensões possuem opções de resposta em escala Likert de 7 pontos, com variância entre discordo totalmente e concordo totalmente. Nessa linha, o Quadro 1 apresenta as dimensões e os indicadores utilizados neste estudo. 

DIMENSÕES E INDICADORES DE CULTURA (HOFSTEDE, 1980)

\begin{tabular}{|c|c|c|}
\hline \multirow{2}{*}{\multicolumn{2}{|c|}{$\begin{array}{c}\text { DIMENSÕES E INDICADORES DE CULTURA (HOFSTEDE, 1980) } \\
\text { DISTÂNCIA DO PODER }\end{array}$}} & \\
\hline & & Confiabilidade \\
\hline \multicolumn{2}{|r|}{ Fatores que compõem a dimensão } & \\
\hline Q1 & $\begin{array}{l}\text { Em sua casa, seus pais devem tomar a maioria das decisões sem } \\
\text { consultar você; }\end{array}$ & 0,62 \\
\hline Q2 & Eu não devo discordar das decisões dos meus pais; & 0,65 \\
\hline Q3 & $\begin{array}{l}\text { Nossa família deve evitar se envolver com pessoas de outra classe } \\
\text { social. }\end{array}$ & 0,66 \\
\hline \multicolumn{2}{|r|}{ COLETIVISMO } & \\
\hline Indicadores & Fatores que compõem a dimensão & \\
\hline Q4 & $\begin{array}{l}\text { Eu devo esquecer (abrir mão) do que quero se isto prejudicar meu } \\
\text { grupo de estudos (trabalhos de escola); }\end{array}$ & 0,60 \\
\hline Q5 & $\begin{array}{l}\text { Eu devo renunciar aos meus próprios interesses em benefício dos } \\
\text { interesses do meu grupo de estudos (trabalhos de escola); }\end{array}$ & 0,66 \\
\hline Q6 & $\begin{array}{l}\text { Eu devo ficar meu grupo de estudos (trabalhos de escola) mesmo } \\
\text { quando ele estiver em dificuldades; }\end{array}$ & 0,61 \\
\hline Q7 & $\begin{array}{l}\text { O bem-estar do meu grupo de estudos (trabalhos de escola) é mais } \\
\text { importante do que as recompensas individuais que eu possa receber; }\end{array}$ & 0,62 \\
\hline Q8 & $\begin{array}{l}\text { O sucesso do meu grupo de estudos (trabalhos de escola) é mais } \\
\text { importante que o meu sucesso individual; }\end{array}$ & 0,64 \\
\hline Q9 & $\begin{array}{l}\text { Só devo perseguir meus objetivos depois de considerar o que é } \\
\text { melhor para o meu grupo de estudos (trabalhos de escola). }\end{array}$ & 0,68 \\
\hline \multicolumn{2}{|c|}{ DIMENSÕES E INDICADORES DE INOVATIVIDADE (PARASURAMANN, 2000) } & \\
\hline \multicolumn{2}{|c|}{ INOVATIVIDADE } & \\
\hline \multicolumn{2}{|r|}{ Fatores que compõem a dimensão } & \\
\hline Q10 & $\begin{array}{l}\text { Seus colegas me procuram para pedir conselhos sobre novidades } \\
\text { em: telefones celulares, tablets, aplicativos, computadores, jogos } \\
\text { eletrônicos e outras tecnologias; }\end{array}$ & 0,62 \\
\hline Q11 & $\begin{array}{l}\text { Parece que seus amigos estão aprendendo mais novidades em: } \\
\text { telefones celulares, tablets, aplicativos, computadores, jogos } \\
\text { eletrônicos e outras tecnologias do que você; }\end{array}$ & 0,63 \\
\hline Q12 & $\begin{array}{l}\text { Em geral, você está entre os primeiros entre seus amigos em } \\
\text { conhecer novidades em: telefone celulares, tablets, aplicativos, } \\
\text { computadores, jogos eletrônicos e outras tecnologias; }\end{array}$ & 0,67 \\
\hline Q13 & $\begin{array}{l}\text { Normalmente, você consegue descobrir novidades em: telefones } \\
\text { celulares, tablets, aplicativos, computadores, jogos eletrônicos e } \\
\text { outras tecnologias, sem a ajuda de outras pessoas; }\end{array}$ & 0,61 \\
\hline Q14 & $\begin{array}{l}\text { Você acompanha novidades em: telefones celulares, tablets, } \\
\text { aplicativos, computadores, jogos eletrônicos e outras tecnologias que } \\
\text { mais lhe interessam; }\end{array}$ & 0,65 \\
\hline Q15 & $\begin{array}{l}\text { Você se diverte com o desafio de descobrir novidades em: telefones } \\
\text { celulares, tablets, aplicativos, computadores, jogos eletrônicos e } \\
\text { outras tecnologias; }\end{array}$ & 0,63 \\
\hline Q16 & $\begin{array}{l}\text { Você acha que tem menos problemas do que outras pessoas em usar } \\
\text { novidades em: telefones celulares, tablets, aplicativos, computadores, } \\
\text { jogos eletrônicos e outras tecnologias; }\end{array}$ & 0,69 \\
\hline Q17 & $\begin{array}{l}\text { Você evitou experimentar novidades em: telefone celulares, tablets, } \\
\text { aplicativos, computadores, jogos eletrônicos e outras tecnologias, por } \\
\text { causa do tempo que leva para aprendê-las; }\end{array}$ & 0,63 \\
\hline Q18 & $\begin{array}{l}\text { Você sempre está aberto a aprender sobre novidades em: telefones } \\
\text { celulares, tablets, aplicativos, computadores, jogos eletrônicos e } \\
\text { outras tecnologias; }\end{array}$ & 0,65 \\
\hline Q19 & $\begin{array}{l}\text { Não há sentido em experimentar novidades em: telefones celulares, } \\
\text { tablets, aplicativos, computadores, jogos eletrônicos e outras } \\
\text { tecnologias, quando o que você já tem está funcionando bem. }\end{array}$ & 0,63 \\
\hline & & \\
\hline
\end{tabular}

FONTE: Adaptado de Hofstede (1980) e Parasuramann (2000).

Os dados levantados por meio da aplicação do questionário foram tabulados em planilha do software Microsoft Exce ${ }^{\circledR}$. As questões foram agrupadas às referidas dimensões, conforme descritas no Quadro 1. A base de dados da planilha do 
software Microsoft Exce ${ }^{\circledR}$ foi importada para o Software Estatístico SPSS ${ }^{\circledR}$ (Statistical Package for the Social Sciences) versão 22.

A primeira análise realizada foi o teste de confiabilidade (Alfa de Cronbach). Este indicador é uma medida de confiabilidade, que varia de 0 a 1 , sendo os valores de 0,60 a 0,70 , considerados o limite inferior de aceitabilidade, conforme apresentado anteriormente no Quadro 1 (HAIR Jr. et al., 2009). Posteriormente, foi realizado uma análise descritiva dos dados, a fim de caracterizar a população investigada neste estudo.

A análise dos dados será realizada por meio das dimensões: distância do poder e coletivismo versus individualismo. Assim, busca-se identificar os elementos da cultura pertencentes ao grupo de adolescentes pertencentes a esta amostra. Por meio das dimensões distância do poder e coletivismo versus individualismo, que podem influenciar na adoção de inovações tecnológicas por adolescentes, utilizouse a técnica de análise de Regressão Linear.

De acordo com Hair Jr. et al. (2009), a regressão linear é a técnica estatística de análise de dados mais aplicada para mensurar relações lineares entre duas ou mais variáveis. É por meio da correlação que se avalia se existe uma relação entre as variáveis do estudo e também qual a força dessa relação, tendo em vista que a regressão linear está diretamente relacionada com a correlação. Para a análise da regressão linear, são necessárias uma variável independente e uma dependente.

Neste estudo, utilizou-se a inovatividade como variável dependente e as questões de cultura como variável independente, cujo interesse era examinar a influência das questões de cultura sobre a variável dependente inovatividade. Os dados foram apresentados em tabelas e textos que culminaram na discussão e análise dos dados apresentados na sequência.

\section{Análise dos Resultados}

Nesta seção é apresentada uma análise descritiva acerca do perfil dos respondentes e uma análise de regressão a fim de verificar se a cultura possui 
influência sobre a inovatividade quanto à aquisição de tecnologia pelos adolescentes.

\section{Análise Descritiva}

Dando sequência ao estudo, a Tabela 1 apresenta os dados referentes aos perfis dos envolvidos na pesquisa, bem como a frequência de respostas deles.

\begin{tabular}{c|c}
\multicolumn{2}{c}{ TABELA $1-$ GÊNERO E IDADE DOS RESPONDENTES } \\
\hline Gênero & Frequência \\
\hline Masculino & 189 \\
\hline Feminino & 279 \\
\hline Outro & 5 \\
\hline Idade & Frequência \\
\hline 14 & $24 \%$ \\
\hline 15 & $27 \%$ \\
\hline 16 & $35 \%$ \\
\hline 17 & $10 \%$ \\
\hline 18 & $2 \%$ \\
\hline 19 & $0,4 \%$ \\
\hline
\end{tabular}

FONTE: Os autores (2016).

Como representado na Tabela 1, a maioria, totalizando 279 dos respondentes, são do gênero feminino, representando 59,11\% dos 472 entrevistados. A faixa etária dos estudantes da instituição varia de 14 a 19 anos. Vale ressaltar ainda que a média de idade dos respondentes é de 15 anos.

$\mathrm{Na}$ Tabela 2, a seguir, são indicados os familiares com os quais os respondentes residem.

TABELA 2 - FAMILIARES COM OS QUAIS OS ADOLESCENTES MORAM

\begin{tabular}{c|c}
\hline Mora Com & Frequência \\
\hline Mãe e Pai & 317 \\
\hline Mãe & 118 \\
\hline Pai & 27 \\
\hline Outros & 11 \\
\hline
\end{tabular}

FONTE: Os autores (2016).

Conforme os dados apresentados na Tabela 2, percebe-se que a maioria, $67,16 \%$ dos adolescentes, moram com ambos os pais (317 respondentes), 118 
moram apenas com a mãe e 27 moram com o pai. Dos respondentes, 11 indicaram morar com outras pessoas, fora da família nuclear. Na Tabela 3, os respondentes são classificados com relação à propriedade do imóvel no qual residem.

TABELA 3 - PROPRIEDADE DO IMÓVEL

\begin{tabular}{c|c}
\hline Residência & Frequência \\
\hline Própria & 401 \\
\hline Alugada & 66 \\
\hline Emprestada & 6 \\
\hline Na praia & 156 \\
\hline
\end{tabular}

FONTE: Os autores (2016).

Observa-se que a maioria dos adolescentes, em um total de 84,96\%, residem em casa própria (401 respondentes), 66 adolescentes disseram morar em residência alugada, enquanto 6 indicaram residir em residência emprestada. Além disso, 33\% possuem residência na praia, indicando um padrão que possibilite a aquisição de dois imóveis. Quando questionados se a família possuía carro, 93\% afirmaram que sim. Não foi perguntada a quantidade de carros na família, só possui ou não veículos.

Quando questionados sobre o nível de escolaridade de seus pais, $26 \%$ indicaram que o pai possui graduação e $23 \%$ pós-graduação, somando $49 \%$ com superior completo. $8 \%$ disseram que o pai está cursando uma faculdade. As mães são as que possuem mais cursos de graduação concluídos, com $28 \%$ e $18 \%$ com pós-graduação, estando $11 \%$ cursando uma faculdade. No total, as mães possuem $46 \%$, somando as que possuem graduação e pós-graduação. Estes dados indicam um nível de educação bastante elevado, sendo indicado somente 10\% com Ensino Fundamental e nenhum pai ou mãe analfabetos.

A análise geral dos dados demográficos indica que os jovens, em sua maioria, possuem uma vida estável, com um valor aquisitivo que viabilize estudarem em uma escola particular, possuir pelo menos um imóvel e, na maioria, ter carro para se locomover. Estes dados indicam que os jovens fazem parte da classe $\mathrm{B}$ e $\mathrm{C}$ da população, estando à maioria na classe B. Não se questionou valores monetários por se supor que um adolescente não tem consciência de quanto sua família recebe em dinheiro por mês. Optou-se, portanto, tentar traçar o perfil socioeconômico por meio da indicação de patrimônio e nível educacional da família nuclear. 
$\mathrm{Na}$ análise para indicação do tipo de cultura dos adolescentes, foi utilizada a análise de frequência. As discordâncias indicavam menor Distância de Poder e um maior Individualismo. A amostra composta por estes adolescentes indica que 67\% se mostra com baixa distância do poder. Os jovens indicam que não devem se renderem ao poder dos pais e questionar o posicionamento de seus genitores. No que concerne à dimensão de Coletivismo e Individualismo, não houve uma indicação para caracterizá-los em qualquer uma das categorias. Do total, 39\% se mostram individualista, $38 \%$ coletivista e $23 \%$ nem coletivista, nem individualista.

\section{Análise de Regressão}

Primeiramente, para que fosse possível rodar a análise de regressão linear, com o objetivo de verificar se a cultura possui influência sobre a inovatividade, definiu-se como variável dependente as questões pertencentes à Inovatividade. A análise é composta por dez questões, conforme descritas no Quadro 1, em que foi realizada a função média a fim de representar a variável (MINOVA). As variáveis independentes foram formadas pelas nove questões que pertencem a duas dimensões da cultura: distância do poder e coletivismo, que também podem ser vistas no Quadro 1.

Em seguida, foi realizada uma análise de confiabilidade para verificar 0 alpha de cronbach das questões (variáveis). Conforme Hair Jr et al. (2009), o alfa de Cronbach estima o grau de convergência dos dados. De tal modo, quanto maior for este grau (deve ser maior que 0,6), maior será a confiabilidade das respostas da pesquisa.

Em seguida, foi executada a função de "excluir a variável" que estivesse contribuindo negativamente na consistência interna da dimensão. Sendo assim, foram excluídas as questões: Q11, Q17 e Q19 (pertencentes à dimensão da variável independente inovatividade) e atingiu-se um valor de alpha de cronbach de 0,63.

Na Tabela 4 apresenta-se o índice de significância (Sig.) que as variáveis independentes possuem para o modelo de regressão. Ressalta-se que essas 
variáveis pertencem a cultura, cujas questões dividem-se nas dimensões: distância do poder (Q1, Q2 e Q3) e coletivismo (Q4, Q5, Q6, Q7, Q8, Q9).

TABELA 4 - SIGNIFICÂNCIA (MAD)

\begin{tabular}{|c|c|c|c|c|c|}
\hline \multirow{2}{*}{$\begin{array}{l}\text { Modelo Antes da } \\
\text { Depuração (MAD) }\end{array}$} & \multicolumn{2}{|c|}{ Coeficientes não padronizados } & \multirow{2}{*}{$\begin{array}{c}\text { Coeficientes } \\
\text { padronizados } \\
\text { Beta }\end{array}$} & \multirow[t]{2}{*}{$t$} & \multirow[t]{2}{*}{ Sig. } \\
\hline & $\mathrm{B}$ & Modelo padrão & & & \\
\hline (Constante) & 3,498 &, 175 & & 19,991 &, 000 \\
\hline Q1 &,- 039 & ,026 &,- 074 & $-1,503$ & ,134 \\
\hline Q2 & ,045 & ,024 & ,094 & 1,885 &, 060 \\
\hline Q3 & ,040 &, 030 &, 062 & 1,338 &, 182 \\
\hline Q4 &, 068 & ,022 & , 156 & 3,154 &, 002 \\
\hline Q5 & ,018 &, 024 &, 037 &, 750 &, 454 \\
\hline Q6 &, 023 &, 023 & 048 & 1,003 &, 316 \\
\hline Q7 & 019 & 026 & 041 & .759 & 448 \\
\hline Q8 &, 011 & 024 &, 024 & ,438 & $662^{*}$ \\
\hline Q9 & ,005 & ,024 &, 010 & , 190 &, $850^{\star}$ \\
\hline
\end{tabular}

FONTE: Os autores (2016).

NOTA: * Retiradas do modelo.

De acordo com Hair Jr. et al. (2009), uma variável só é significante estatisticamente se o p-value estiver abaixo de 0,05. Nesse caso, conforme a Tabela 4, a regressão demonstrou que apenas a variável independente Q4 é significante. A variável Q2 apresentou-se marginalmente significante. As variáveis que não foram significantes para o modelo de regressão fazem parte da dimensão: Individualismo e Coletivismo. Elas estão relacionadas à percepção do respondente quanto à importância que ele atribui ao seu sucesso individual e ao sucesso do grupo. Neste quesito, demonstra-se que os respondentes apresentaram um perfil mais individualista, considerando seus objetivos pessoais mais relevantes do que os objetivos comuns ao seu grupo social.

Segundo Gouveia, Clemente e Vidal (1998), o sujeito individualista valoriza a adesão aos grupos em função de prováveis benefícios. Este perfil sugere que há uma independência em relação aos familiares e desvinculação dos laços parentais. Da mesma forma, Gouveia (2001) afirma que pessoas guiadas pelo individualismo tendem a restringir seus grupos apenas à família nuclear. Suas ações e pensamentos voltam-se primeiramente aos interesses próprios, relegando a um segundo plano, o contexto social no qual se encontram. 
Já Perez-Alvarez (2009) afirma que culturas individualistas se caracterizam pela influência de crenças pessoais na tomada de decisões e nem sempre as regras do grupo são rigorosamente seguidas.

Posteriormente, realizou-se uma depuração no modelo (Tabela 5), em que foram excluídas a variáveis Q8 e Q9, a fim de buscar um ajuste melhor aos índices das demais variáveis. Na Tabela 5 são apresentados os índices após a exclusão dessas variáveis.

TABELA 5 - SIGNIFICÂNCIA (MD)

\begin{tabular}{l}
\multicolumn{2}{c}{$\begin{array}{c}\text { Modelo Depurado } \\
\text { (MD) }\end{array}$} \\
\cline { 2 - 7 }
\end{tabular}

a. Variável dependente: MINOVA

FONTE: Os autores (2016)

Conforme verifica-se na Tabela 5, após a exclusão das variáveis Q8 e Q9, houve melhora no índice de significância das variáveis Q2, Q4, Q5, Q6 e Q7. A dimensão Distância do Poder está representada pelas questões Q1, Q2 e Q3. Na observação destas questões, por meio do nível de significância, observa-se que a Q2 - Não devo discordar das decisões de meus pais - possui uma Sig. de 0,046. Este valor indica que os adolescentes enfrentam os pais quando estes tendem a tomar as decisões sem a participação deles. Pode-se concluir, portanto, que este é o único elemento da distância do poder, indicando que existe uma baixa distância, que está impactando na adoção de inovações tecnológicas.

A dimensão Coletivismo versus Individualismo é representada pelas questões Q4, Q5, Q6 e Q7. Observa-se que o único indicador significante foi a questão Q4 - Eu devo esquecer (abrir mão) do que quero se isto prejudicar meu grupo de estudos (trabalhos de escola) - possui uma Sig. de 0,001. A significância "abrir mão do que se quer para não prejudicar o grupo de estudo" influencia 
positivamente a inovatividade dos adolescentes pertencentes a esta amostra. $\mathrm{Na}$ Tabela 6, são apresentadas as estatísticas de colinearidade, por meio da tolerância e do fator de inflação da variância (VIF).

TABELA 6 - ESTATÍSTICAS DE COLINEARIDADE

\begin{tabular}{c|c|c|c|c|c}
\hline \multirow{2}{*}{$\begin{array}{c}\text { Modelo Antes da } \\
\text { Depuração } \\
\text { (MAD) }\end{array}$} & $\begin{array}{c}\text { Coeficientes } \\
\text { padronizados }\end{array}$ & \multicolumn{2}{|c|}{$\begin{array}{c}\text { Estatísticas de } \\
\text { colinearidade }\end{array}$} & \multicolumn{2}{c}{ Modelo Depurado (MD) } \\
\cline { 2 - 6 } & Beta & Tolerância & VIF & \multicolumn{2}{c}{ Estatísticas de colinearidade } \\
\cline { 5 - 6 } & & & & & Tolerância \\
\hline Constante & &, 833 & 1,201 &, 834 & 1,199 \\
\hline Q1 &, 156 &, 817 & 1,224 &, 846 & 1,183 \\
\hline Q2 &,- 074 &, 948 & 1,055 &, 952 & 1,050 \\
\hline Q3 &, 094 &, 836 & 1,196 &, 849 & 1,178 \\
\hline Q4 &, 062 &, 840 & 1,190 &, 854 & 1,171 \\
\hline Q5 &, 037 &, 883 & 1,132 &, 883 & 1,132 \\
\hline Q6 &, 048 &, 714 & 1,400 &, 861 & 1,162 \\
\hline Q7 &, 041 &, 698 & 1,432 & Var. Excluída & Var. Excluída \\
\hline Q8 &, 024 &, 752 & 1,330 & Var. Excluída & Var. Excluída \\
\hline Q9 &, 010 & &
\end{tabular}

FONTE: Os autores (2016)

Segundo Hair Jr. et al. (2009), o fator de inflação da variância (VIF) mede o efeito das variáveis independentes sobre o coeficiente de regressão. O VIF está relacionado de forma inversa ao valor de tolerância. Quando os valores de VIF são altos, indica alto grau de colinearidade ou multicolinearidade entre as variáveis independentes, ou seja, fator indesejável. A colinearidade é a relação entre duas variáveis ou mais (multicolinearidade). As variáveis apresentam uma colinearidade completa se seu coeficiente de correlação for 1 e falta de colinearidade quando mais próximo de 0 , sendo 5 considerado satisfatório. Quanto ao índice de tolerância, segundo Hair Jr. et al. (2009), devem estar o mais próximo de 1.

Assim, conforme apresentado na Tabela 6, quando realizado o teste de colinearidade por meio do fator de inflação da variância, verificou-se que todas as variáveis estavam abaixo de 5 , o que demonstra baixa correlação entre as variáveis independentes e todos os valores de tolerância próximas de 1. Estes índices denotam um resultado positivo, pois demonstram que não há colinearidade entre as variáveis independentes.

Devido à Sig. das variáveis Q8 e Q9 não estarem dentro dos parâmetros exigidos, rodou-se novamente o modelo, excluindo essas variáveis. Assim, pôde-se

GONÇALVES, A.; TAMBOSI, S. V.; MONDINI, V.; MACHADO, D. D. P. N.O impacto da cultura no uso de produtos com tecnologia agregada: estudo com adolescentes. Revista de Empreendedorismo e Gestão de Pequenas Empresas, v.5, n.1, 2016. 
constatar (Tabela 6) que o modelo estatístico de colinearidade apresentou melhores índices de tolerância e de fator de inflação da variância (VIF) das variáveis: Q3 (Tolerância: 0,948 e o VIF: 1,050); em seguida a Q6 (Tolerância: 0,883 e o VIF: 1,132); Q7 (Tolerância: 0,861 e o VIF: 1,162); Q5 (Tolerância: 0,854 e o VIF: 1,171); Q4 (Tolerância: 0,849 e o VIF: 1,178); Q1 (Tolerância: 0,834 e o VIF: 1,199); Q2 (Tolerância: 0,846 e o VIF: 1,183).

Verifica-se que a variável Q3, da cultura que pertence a dimensão "distância do poder", foi a que alcançou melhores índices, tanto antes quanto depois do ajuste do modelo de regressão. Entretanto, a dimensão "coletividade" também apresentou um bom conjunto de valores (maior número de variáveis). Na Tabela 7 é apresentada a correlação entre a variável dependente com as independentes $(R)$ e o seu grau de explicação $\left(R^{2}\right)$.

\begin{tabular}{|c|c|c|c|}
\hline \multicolumn{2}{|c|}{$\begin{array}{c}\text { Modelo } \\
\text { Antes da Depuração } \\
\text { (MAD) }\end{array}$} & \multicolumn{2}{|c|}{$\begin{array}{l}\text { Modelo } \\
\text { Depurado } \\
\text { (MD) }\end{array}$} \\
\hline $\mathrm{R}$ & $\mathrm{R}^{2}$ & $\mathrm{R}$ & $\mathrm{R}^{2}$ \\
\hline ,241 & ,058 & ,241a & ,058 \\
\hline
\end{tabular}

FONTE: Os autores (2016).

NOTA: a. Preditores: (Constante), Q1, Q2, Q3, Q4, Q5, Q6, Q7, Q8, Q9

Por meio da Tabela 7, é possível verificar que a correlação foi de $24,1 \%$ entre a variável dependente (Inovatividade) com as independentes (Distância do Poder: Q1, Q2, Q3 e Coletivismo: Q4, Q5, Q6, Q7, Q8, Q9) e o grau de explicação $\left(R^{2}\right)$ dessas variáveis foi de $5,8 \%$, esse índice representa o grau de determinação. Pode-se perceber que 0 índice foi baixo. Isso significa que as variáveis independentes só explicam $5,8 \%$ da variação da variável independente.

Segundo Koufteros (1999), um nível adequado de $\mathrm{R}^{2}$ deve ser acima de 0,5. Diante disto, constata-se que o grau de determinação desta pesquisa é aceitável. É possível constatar que mesmo com a exclusão das variáveis “Q8 e Q9", não houve alteração nos índices do modelo de $\mathrm{R}$ e $\mathrm{R}^{2}$. 


\section{Considerações Finais}

Este estudo buscou identificar elementos da cultura, por meio das dimensões distância do poder e coletivismo versus individualismo, que podem influenciar na adoção de inovações tecnológicas por adolescentes de uma escola particular no Vale do Itajaí. Foram aplicados questionários com perguntas estruturadas em escala Likert de sete pontos, em 472 adolescentes com idade média de 15 anos.

Os resultados apresentados por este estudo suscitaram algumas divergências quanto ao encontrado nos trabalhos de Hofstede (1980). O autor apresenta a sociedade brasileira como coletivista e com alta distância do poder. Os adolescentes participantes nesta pesquisa, indicam uma tendência ao individualismo e à baixa distância do poder. Convém ressaltar que o aspecto medido nesta pesquisa se refere à adoção de inovações tecnológicas e os relacionamentos com os pais e colegas. A distância do poder foi medida por meio da percepção do adolescente quanto ao enfrentamento com seus pais.

Em um trabalho sobre tomada de decisão em consumo na família, feito por Shoham e Dalakas (2005), os autores identificam que, adolescentes com maior distância do poder da cultura Israelense, tendem a ser mais rebeldes e enfrentarem os pais do que adolescentes americanos. É preciso lembrar que a distância do poder em Israel é maior do que nos Estados Unidos.

A dimensão coletivismo versus individualismo foi identificada por meio do trabalho em grupos escolares. As respostas indicam que os adolescentes tendem a ser individualistas e trocarem os ganhos em grupo por ganhos próprios. Estes dados divergem da análise feita por Hofstede (1980) sobre a cultura brasileira. Os brasileiros tendem a ser coletivista e priorizarem o grupo, fato que não foi observado na amostra de adolescentes.

Convém ressaltar que Hofstede (1980) desenvolveu sua pesquisa na década de 1970, em uma empresa multinacional, situada em 50 países diferentes. Salientase que desde esta pesquisa já decorreram 40 anos. Nesta época, o Brasil possuía um regime militar e as fronteiras econômicas estavam fechadas, indicando uma 
reserva de mercado para produtos nacionais. Com o avanço das tecnologias de informação, os adolescentes passaram a ter muito mais contato com outras culturas, além de que seus pais não foram criados sob a ditadura militar.

Nesse sentido, pode-se supor que houve mudança nos padrões culturais da população, além de que a presente pesquisa foi feita com indivíduos em uma faixa etária que tem como bandeira o enfrentamento aos pais e o egoísmo próprio da idade. Ressalta-se que, decorrente do distanciamento do regime militar, o "medo" em expressar uma liberdade já se dissipou, tornando os atuais adolescentes menos tolerantes às demonstrações de poder sobre eles próprios.

É importante dizer que este estudo não pretende questionar os resultados apresentados por Hofstede (1980) em seu trabalho, mas lançar novos olhares sobre os futuros profissionais que irão adentrar o mercado de consumo no Brasil. Supõese que as contribuições deste estudo possam auxiliar na compreensão dos elementos da cultura presentes na região na qual o trabalho foi desenvolvido e na parcela da população que tende a abraçar novas tecnologias cada vez mais rápido.

Por fim, propõe-se que este estudo seja replicado com o intuito de averiguar o impacto da cultura sobre a adoção de produtos inovadores, averiguando também a velocidade e liderança do jovem na adoção de inovações tecnológicas. Entende-se, também, que as limitações estão justamente por ser este, um estudo exploratório e os resultados não podem ser generalizados para outras regiões do Brasil, principalmente por ser um país com dimensões continentais. Sendo assim, recomenda-se a replicação deste trabalho em diferentes faixas etárias e outras regiões do Brasil para que se possa comparar os resultados.

\section{Referências:}

AFUAH, A. Innovation management: strategies, implementation and profits. Oxford University Press: New York, 2003.

AGARWAL, R.; PRASAD, J. A Conceptual and Operational Definition of Personal Innovativeness in the Domain of Information Technology. Information Systems Research, v. 9, n. 2, p. 204-215, 1998.

AKOVA, B.; ULUSOY, G.; PAYZIN, E.; KAYLAN, A. R. As novas capacidades de desenvolvimento de produtos da indústria eletrônica turcos. In: V CONFERÊNCIA 
INTERNACIONAL DE GESTÃO DE DESENVOLVIMENTO DE PRODUTO, Anais... Como, Itália, 1998. p. 863-876.

ALEGRE, J.; LAPIEDRA, R.; CHIVA, R. A measurement scale for product innovation performance. European Journal of Innovation Management, v. 9, n. 4, p. 333346, 2006.

BROWN, S. L.; EISENHARDT, K. M. Product development: past research, present findings, and future directions. Academy of Management Review, v. 20, n. 2, p. 343-378, 1995.

CHANDY, R. K.; PRABHU, J. C. Innovation typologies. Wiley international encyclopedia of marketing, 2010.

CHAO, C. W.; REID, M.; MAVONDO, F. The influence of consumer innovativeness on really new product adoption. Anzmac, 2009.

CLARK, R. A; GOLDSMITH, R. E. Interpersonal Influence and Consumer Innovativeness. International Journal of Consumer Studies, v. 30, p. 34-43, 2006.

COOPER, R. G.; KLEINSCHMIDT, E. J. Major new products; what distinguish hes the winners in the chemical industry?. Journal of Product Innovation Management, v. 10, p. 90-111, 1993.

DAGHFOUS, N.; PETROF, J. V.; PONS, F. Values and adoption of innovations: a cross-cultural study. Journal of Consumer Marketing, v. 16, n. 4, p. 314-331, 1999.

DAMANPOUR, F. Organizational Size and Innovation. Organization Studies. v. 13, n. 3, p. 375-402, 1992.

EASTLICK, M. A.; LOTZ, S. Profiling Potential Adopters and Non-Adopters of an Interactive Electronic Shopping Medium. International Journal of Retail \& Distribution Management, v. 27, n. 6, p. 209-223, 1999.

GOLDSMITH, R. E.; HOFACKER, C. F. Measuring Consumer Innovativeness. Journal of the Academy of Marketing Science, v. 19, n. 3, p. 209-221, 1991.

GOPALAKRISHNAN, S.; DAMANPOUR, F. A review of innovation research in economics, sociology and technology management. Omega, v. 25, n. 1, p. 15-28, 1997.

GOUVEIA, V. V.; CLEMENTE, M.; VIDAL, M. A. España desde dentro: El individualismo y el colectivismo como rasgos diferenciadores de las comunidades autónomas. Sociedade y Utopia, v.11, p. 168-179, 1998.

GOUVEIA, V. V. El individualismo y el colectivismo normativo: Comparación de dos modelos. Em M. Ros \& V. V. Gouveia (Orgs.), Psicología social de los valores

GONÇALVES, A.; TAMBOSI, S. V.; MONDINI, V.; MACHADO, D. D. P. N.O impacto da cultura no uso de produtos com tecnologia agregada: estudo com adolescentes. Revista de Empreendedorismo e Gestão de Pequenas Empresas, v.5, n.1, 2016. 
humanos: Desarrollos teóricos, metodológicos y aplicados, p. 101-122. Madri: Editorial Biblioteca Nueva, 2001.

GRIFFIN, A. PDMA research on new product development practices: updating trends and benchmarking best practices. Journal of Product Innovation Management, v. 14, n. 6, p. 429-458, 1997.

HAIR Jr. et al. Análise Multivariada de Dados. 6. ed. Porto Alegre: Bookman, 2009.

HENDERSON, R. M.; CLARK, K. B. Architectural innovation: the reconfiguration of existing product technologies and the failure of established firms. Administrative Science Quarterly, p. 9-30, 1990.

HOFSTEDE, G. Culture's consequences: international differences in work-related values. Beverly Hills, CA: Sage, 1980.

HURLEY, R. F.; HULT, G. T. M. Innovation, market orientation, and organizational learning: an integration and empirical examination. J Mark, v. 62, n. 3, p. $42-54$, 1998.

HURT, H. T.; JOSEPH, K.; COOK, C. D. Scale for the Measurement of Innovativeness. Human Communication Research, v. 4, n. 1, p. 58-65, 1977.

KALLINY, M.; HAUSMAN, A. The impact of cultural and religious values on consumer's adoption of innovation. Academy of Marketing Studies Journal, v. 11, n. 1, p. 125-136, 2007.

KOUFTEROS, X. A. Testing a model of pull production: a paradigm for manufacturing research using structural equation modeling. Journal of Operations Management, v. 17, n. 4, p. 467-488, 1999.

MCCRINDLE, M. The ABC of the XYZ: Understanding global Generations. Sydney: UNSW Press, 2011.

MIDGLEY, D. F.; DOWLING, G. R. Innovativeness: the concept and its measurement. Journal of Consumer Research, p. 229-242, 1978.

OECD. Manual de Oslo: diretrizes propostas para a coleta e interpretação de dados da inovação tecnológica. Paris, 2005.

PARASURAMAN, A. Technology Readiness Index (TRI) a multiple-item scale to measure readiness to embrace new technologies. Journal of Service Research, v. 2, n. 4, p. 307-320, 2000.

PEREZ-ALVAREZ, C. How Uncertainty Avoidance Impacts Groupware Appropriation. Proceedings of the Academy of Information and Management Sciences Conference, v. 13, n. 1, p. 43-50, 2009

GONÇALVES, A.; TAMBOSI, S. V.; MONDINI, V.; MACHADO, D. D. P. N.O impacto da cultura no uso de produtos com tecnologia agregada: estudo com adolescentes. Revista de Empreendedorismo e Gestão de Pequenas Empresas, v.5, n.1, 2016. 
PETTIGREW, A. M. On studying organizational cultures. Administrative Science Quarterly, p. 570-581, 1979.

ROEHRICH, G. Consumer innovativeness Concepts and measurements. Journal of Business Research, v. 57, n. 6, p. 671-677, 2004.

ROSEN, P. A. The effect of personal innovativeness on technology acceptance and use.85 f. Tese (Doutorado em Filosofia). Oklahoma State University, 2005.

SCHUMPETER, J. A. A Teoria do Desenvolvimento Econômico: uma investigação sobre lucros, capital, crédito, juro e ciclo de negócios. Harvard University Press: Cambridge, 1934.

SHOHAM, A.; DALAKAS, V. He said, she said... they said: parents' and children's assessment of children's influence on family consumption decisions. Journal of Consumer Marketing, v. 22, n. 3, p. 152-160, 2005.

STEENKAMP, J. B. E. M; HOFSTEDE, F.; WEDEL, M. A cross-national comparison in to the national and national cultural antecedents of consumer inovativeness. $J$ Mark, v. 63, n. 2, p. $55-69,1999$.

TOLBA, A. H.; MOURAD, M. Individual and cultural factors affecting diffusion of innovation. Journal of International Business and Cultural Studies, v. 5, n. 3, p. 203-218, 2011.

TRIANDIS, H. C. Individualism \& collectivism. Westview Press, 1995.

TUSHMAN, M. L. et al. Organizational determinants of technological change: Towards a sociology or technology evolution. Research in Organizational Behavior, v. 14, p. 311-347, 1992. 